Annals of Pure and Applied Mathematics

Vol. 16, No. 2, 2018, 393-400

ISSN: 2279-087X (P), 2279-0888(online)

Published on 3 March 2018

www.researchmathsci.org

DOI: http://dx.doi.org/10.22457/apam.v16n2a16

Annals of

Pure and Applied

Mathematics

\title{
On $p$ - $h$ Points and Completeness Property of a Partial Metric Space
}

\author{
A.P.Baisnab ${ }^{1}$ and Sumana Pal ${ }^{2}$ \\ ${ }^{1}$ Department of Mathematics, Lady Brabourne College \\ Kolkata -700017, West Bengal, India. E-mail: baisnababhoypada@yahoo.com \\ ${ }^{2}$ Department of Mathematics, Aliah University \\ Kolkata-700156, West Bengal, India. E-mail: sumana.pal@gmail.com \\ ${ }^{2}$ Corresponding author
}

Received 10 February 2018; accepted 28 February 2018

\begin{abstract}
Role of $d$-points in a metric space is well-known. The notion has been extended in a partial metric space $(X, p)$ and the consequences of $p$ - $h$ points have been investigated with some applications in theory of fixed points.
\end{abstract}

Keywords: Partial metric spaces, completeness, $p$ - $h$ points, fixed point.

AMS Mathematics Subject Classification (2010): 47H10

\section{Introduction}

The notion of partial metric spaces, which allow non-zero self-distance, was introduced as a generalization of metric spaces by Matthews [14] in 1994 where he gave a generalization of Banach's contraction mapping theorem over a metric space. Since then, many researchers have worked on different aspects, in particular, in the realm of fixed point theory of partial metric spaces. For investigations in partial metric spaces, works of Valero and Oltra [9, 15], Romaguera [16], Altun et al. [6], Choudhury [2, 3] are noteworthy. In this connection it should also be mentioned that before the introduction of partial metric spaces, there were other generalizations of metric spaces, most notably 2metric spaces and generalized metric spaces where fixed point theorems for contractive mappings had been investigated (see for example the work of Das et al. $[4,10]$ or Dey et al. [17]. Again in [1, 5, 11] one finds several fixed point theorems proved for operators involving Kannan contractions, weak contractions and ground space sometimes endowed with an associated graph. In particular, exclusively with Kannan contraction in fixed point theory, we have references like $[8,12,13]$.

In the year 1977, Weston [7] defined $d$-point for a real-valued functionhon a metric space $(X, d)$ and obtained a characterization of completeness ofthe metric space $(X, d)$. Further, he applied the result in fixed point theory over a complete metric space $(X, d)$.

In any generalization of metric spaces, completeness has always been one of the most fundamental properties. In particular characterizations of completeness has been of much interest. In this paper, we have defined $p-h$ point in the setting of partial metric 


\section{A.P. Baisnab and Sumana Pal}

spacesand studied the completeness of a partial metric space with the aid of such $p$ - $h$ points. Also, the concept of $p-h$ points was utilized to deduce some fixed point theorems over complete metric spaces.

We recall a few definitions first.

A partial metric on $X$ is a function $p: X \times X \rightarrow \mathbb{R}^{+}$(the set of nonnegative real numbers) satisfying conditions as under:

For all $x, y, z \in X$,

(i) $\quad x=y$ if and only if $p(x, x)=p(y, y)=p(x, y)$,

(ii) $\quad p(x, x) \leq p(x, y)$,

(iii) $\quad p(x, y)=p(y, x)$,

(iv) $\quad p(x, z) \leq p(x, y)+p(y, z)-p(y, y)$.

If $p$ is a partial metric on the set $X,(X, p)$ is known as a partial metricspace. Thus in a partial metric space $(X, p)$, each point does not necessarily possess zero distance from itself. Of course, a metric space is a partial metric space while the converse is false.

Example 1.1. Take $\mathbb{N}=$ set of all natural numbers, and $p: \mathbb{N} \times \mathbb{N} \rightarrow \mathbb{R}^{+}$is defined by

Then $(\mathbb{N}, p)$ is a partial metric space.

$$
p(m, n)=\left\{\begin{array}{c}
\frac{1}{n} \text { if } m=n, \\
\frac{1}{n}+\frac{1}{m} \text { if } m \neq n .
\end{array}\right.
$$

Now let us give a look into the topological aspects of a partial metric space $(X, p)$.

If $x \in X$ and $\epsilon>0$, then the set $B_{\epsilon}(x)=\{y \in X: p(x, y)<p(x, x)+\epsilon\}$ is called a $p$ open ball in $(X, p)$. By routine check up one finds $\left\{B_{\epsilon}(x)\right\}, x \in X$ and $\epsilon>0$ is a base to generate a topology $\tau_{p}$ called the partial metric topologyon $X$, and this topology $\tau_{p}$ is $T_{0}$.

We have the following definitions in a partial metric space $(X, p)$.

Definition 1.2. A sequence $\left\{x_{n}\right\}$ in a partial metric space $(X, p)$ is said tobe a $p$-Cauchy sequence if $\lim _{m, n \rightarrow \infty} p\left(x_{n}, x_{m}\right)$ exists.

Definition 1.3. A sequence $\left\{x_{n}\right\}$ in a partial metric space $(X, p)$ is said tobe $p$-convergent at $x_{0} \in X$ if $\lim _{n \rightarrow \infty} p\left(x_{n}, x_{0}\right)=p\left(x_{0}, x_{0}\right)$.

Definition 1.4. A partial metric space $(X, p)$ is said to be complete if every $p$-Cauchy sequence in $(X, p) p$-converges to a point of $X$, i.e., if $\left\{x_{n}\right\}$ is $p$-Cauchy in $(X, p)$, there is a point $x_{0} \in X$ such that

$$
\lim _{n, m \rightarrow \infty} p\left(x_{n}, x_{m}\right)=\lim _{n \rightarrow \infty} p\left(x_{n}, x_{0}\right)=p\left(x_{0}, x_{0}\right) .
$$

\section{Main results}

Before going into the main result, we give some basic definitions. 
On $p$ - $h$ Points and Completeness Property of a Partial Metric Space

Definition 2.1. A function $h:(X, p) \rightarrow \mathbb{R}$ (with usual topology) is said to be $p$-lower semi continuous (p-1.s.c.) at $u \in X$ if given $\epsilon>0$, there is a $\delta>0$ such that

$$
h(x)>h(u)-\epsilon \text { for } x \in p-B_{\delta}(u)
$$

or, equivalently, if $\left\{x_{n}\right\}$ is a sequence in $(X, p) p$-converging to $u$, then

$$
\varliminf_{n \rightarrow \infty} f\left(x_{n}\right) \geq f(u) \text {. }
$$

Also $h$ is said to be a $p$-l.s.c. function on $X$ if it is so at every point of $X$.

Definition 2.2. Given a function $h:(X, p) \rightarrow \mathbb{R}$ (with usual topology) a point $x_{0} \in X$ is said to be a $p$ - $h$ point if and only if for every $x \in X$ with $x \neq x_{0}$,

$$
h\left(x_{0}\right)-h(x)<p\left(x_{0}, x\right)-p(x, x)(>0) .
$$

Theorem 2.3. Let $(X, p)$ be a complete partial metric space and $h:(X, p) \rightarrow \mathbb{R}$ be a $p$ lower semi continuous function that is bounded below, then there is a $p-h$ point in $X$.

Proof: Let $h:(X, p) \rightarrow \mathbb{R}$ be a $p$-lower semi continuous function that is bounded below where $(X, p)$ is complete. We consider a member $x_{1} \in X$; let $x_{1}$ be not a $p$ - $h$ point for $h$ in $X$. Then we can find $x \in X$ with $x \neq x_{1}$ satisfying

$$
h\left(x_{1}\right)-h(x) \geq p\left(x_{1}, x\right)-p(x, x) \quad(>0) .
$$

We construct a sequence $\left\{x_{n}\right\}$ in $X$ such that for each $n$, if

$c_{n}=\inf \left\{h(x): h\left(x_{n}\right)-h(x) \geq p\left(x_{n}, x\right)-p(x, x)>0\right\}$,

then $x_{n+1} \in X$ satisfies

and $h\left(x_{n+1}\right)<c_{n}+\frac{1}{n}$.

$$
h\left(x_{n}\right)-h\left(x_{n+1}\right) \geq p\left(x_{n}, x_{n+1}\right)-p\left(x_{n+1}, x_{n+1}\right)
$$

If some $x_{n}$ is a $p$ - $h$ point we are done; then $x_{n+1}=x_{n}$ or else (2.1) shows $\left\{h\left(x_{n}\right)\right\}$ is monotonic decreasing. Suppose $m>n$. Then we have

$$
\begin{aligned}
& h\left(x_{n}\right)-h\left(x_{m}\right) \\
& =\left(h\left(x_{n}\right)-h\left(x_{n+1}\right)\right)+\left(h\left(x_{n+1}\right)-h\left(x_{n+2}\right)\right)+\cdots+\left(h\left(x_{m-1}\right)-h\left(x_{m}\right)\right) \\
& \geq\left(p\left(x_{n}, x_{n+1}\right)-p\left(x_{n+1}, x_{n+1}\right)\right)+\left(p\left(x_{n+1}, x_{n+2}\right)-p\left(x_{n+2}, x_{n+2}\right)\right) \\
& \quad+\cdots+\left(p\left(x_{m-1}, x_{m}\right)-p\left(x_{m}, x_{m}\right)\right) \\
& \geq\left(p\left(x_{n}, x_{n+2}\right)-p\left(x_{n+2}, x_{n+2}\right)\right)+\left(p\left(x_{n+2}, x_{n+3}\right)-p\left(x_{n+3}, x_{n+3}\right)\right) \\
& \quad+\cdots+\left(p\left(x_{m-1}, x_{m}\right)-p\left(x_{m}, x_{m}\right)\right) \\
& \geq\left(p\left(x_{n}, x_{n+3}\right)-p\left(x_{n+3}, x_{n+3}\right)\right)+\cdots+\left(p\left(x_{m-1}, x_{m}\right)-p\left(x_{m}, x_{m}\right)\right) \\
& \geq p\left(x_{n}, x_{m}\right)-p\left(x_{m}, x_{m}\right)>0 .
\end{aligned}
$$

Thus

$h\left(x_{n}\right)-h\left(x_{m}\right) \geq p\left(x_{n}, x_{m}\right)-p\left(x_{m}, x_{m}\right)$.

As $h$ is bounded and $\left\{h\left(x_{n}\right)\right\}$ is monotonic decreasing, $\left\{h\left(x_{n}\right)\right\}$ is convergent and (2.3) says $\left\{x_{n}\right\}$ is $p$-convergent in the partial metric space $(X, p)$.

Let $p$ - $\lim _{n \rightarrow \infty} x_{n}=x_{0} \in X$.

Then we have

Now

$$
\lim _{n, m \rightarrow \infty} p\left(x_{n}, x_{m}\right)=\lim _{n \rightarrow \infty} p\left(x_{n}, x_{0}\right)=p\left(x_{0}, x_{0}\right) .
$$

$h\left(x_{n}\right)-h\left(x_{0}\right) \geq p\left(x_{n}, x_{0}\right)-p\left(x_{0}, x_{0}\right)$. 


\section{A.P. Baisnab and Sumana Pal}

This is true for all $n$.

Otherwise for some $n$, we have

$h\left(x_{n}\right)-h\left(x_{0}\right)<p\left(x_{n}, x_{0}\right)-p\left(x_{0}, x_{0}\right)$.

Choose $\epsilon>0$. Then

$$
h\left(x_{n}\right)-h\left(x_{0}\right)<p\left(x_{n}, x_{0}\right)-p\left(x_{0}, x_{0}\right)-\epsilon .
$$

Let $\epsilon_{1}=p\left(x_{n}, x_{0}\right)-p\left(x_{0}, x_{0}\right)-\epsilon-h\left(x_{n}\right)+h\left(x_{0}\right)(>0)$. We apply $p$-l.s.c. of $h$ at $x_{0}$ to get

$$
h\left(x_{0}\right)-\epsilon_{1}<h(x), \text { for all } x \in p-B_{\delta}\left(x_{0}\right) \text { for some } \delta>0,
$$

$$
\text { or, } h\left(x_{0}\right)-\left\{p\left(x_{n}, x_{0}\right)-p\left(x_{0}, x_{0}\right)-\epsilon-h\left(x_{n}\right)+h\left(x_{0}\right)\right\}<h(x),
$$

or, $h\left(x_{n}\right)-h(x)<p\left(x_{n}, x_{0}\right)-p\left(x_{0}, x_{0}\right)-\epsilon$.

As $\left\{x_{n}\right\} \rightarrow x_{0}$, for large $m, x_{m} \in p-B_{\delta}\left(x_{0}\right)$, so

$$
\begin{gathered}
h\left(x_{n}\right)-h\left(x_{m}\right)<p\left(x_{n}, x_{0}\right)-p\left(x_{0}, x_{0}\right)-\epsilon \\
<p\left(x_{n}, x_{m}\right)+p\left(x_{m}, x_{0}\right)-p\left(x_{m}, x_{m}\right)-p\left(x_{0}, x_{0}\right)-\epsilon .
\end{gathered}
$$

Taking $\delta=\epsilon$, we have

$$
h\left(x_{n}\right)-h\left(x_{m}\right)<p\left(x_{n}, x_{m}\right)-p\left(x_{m}, x_{m}\right) .
$$

This contradicts (2.3) above. Therefore, for all $n,(2.4)$ holds.

We now claim that $x_{0}$ is a $p$-hpoint for $h$. Otherwise, for some $x$,

$$
h\left(x_{0}\right)-h(x) \geq p\left(x_{0}, x\right)-p(x, x)>0 .(2.5)
$$

Replacing $n$ by $n+1$ in (2.4), we find

Therefore

$$
h\left(x_{n+1}\right)-h\left(x_{0}\right) \geq p\left(x_{n+1}, x_{0}\right)-p\left(x_{0}, x_{0}\right) \text {. }
$$

$=h\left(x_{n+1}\right)+h(x)-h\left(x_{0}\right)$

$$
h(x) \leq h(x)+h\left(x_{n+1}\right)-h\left(x_{0}\right)
$$

$<c_{n}+\frac{1}{n}+h(x)-h\left(x_{0}\right)$.

As $h(x)-h\left(x_{0}\right)$ is negative from (2.5), taking large $n$, we have $h(x)<c_{n}$, which contradicts (2.1). Hence the proof is done.

Now as we look for the converse of Theorem 2.3, we have Theorem 2.5 below in this connection.

Definition 2.4. Let $(X, p)$ be a partial metric space. A function $h:(X, p) \rightarrow \mathbb{R}$ is said to be uniformly continuous on $X$ if given $\epsilon>0$, there exists $\delta=\delta(\epsilon)>0$ such that

$$
|h(x)-h(y)|<\epsilon
$$

whenever $x, y \in X$ and $p(x, y)-\min \{p(x, x), p(y, y)\}<\delta$.

Theorem 2.5. Let $(X, p)$ be a partial metric space which is not complete. Then there exists a uniformly continuous function $h:(X, p) \rightarrow \mathbb{R}$ which isbounded below and has no $p$ - $h$ point in $X$.

Proof. Suppose the partial metric space $(X, p)$ is not complete and let $\left\{x_{n}\right\}$ be a $p$-Cauchy sequence in $X$ which is not $p$-convergent. Then $\lim _{m, n \rightarrow \infty} p\left(x_{m}, x_{n}\right)$ is finite.

Let $x \in X$ such that $p\left(x, x_{n}\right) \neq p\left(x_{n}, x_{n}\right)$. We consider the sequence $\left\{2 p\left(x, x_{n}\right)-\right.$ $\left.2 p\left(x_{n}, x_{n}\right)\right\}$ in $\mathbb{R}$.

Since $p\left(x, x_{n}\right) \leq p\left(x, x_{m}\right)+p\left(x_{m}, x_{n}\right)-p\left(x_{m}, x_{m}\right)$, we have $\left\{p\left(x, x_{n}\right)-p\left(x_{n}, x_{n}\right)\right\}-\left\{p\left(x, x_{m}\right)-p\left(x_{m}, x_{m}\right)\right\} \leq p\left(x_{m}, x_{n}\right)-p\left(x_{n}, x_{n}\right) \rightarrow 0$ 
On $p$ - $h$ Points and Completeness Property of a Partial Metric Space as $n \rightarrow \infty$, which shows that $\left\{2 p\left(x, x_{n}\right)-2 p\left(x_{n}, x_{n}\right)\right\}$ is a Cauchy sequence in $\mathbb{R}$. Let $h(x)$ be its limit. Then $h(x)>0$ so that $h$ is bounded below.

Let $x_{0} \in X$. Then

$$
\begin{aligned}
& \left|h\left(x_{0}\right)-h(x)\right|=\left|\lim _{n \rightarrow \infty}\left[2 p\left(x_{0}, x_{n}\right)-2 p\left(x, x_{n}\right)\right]\right| \\
= & 2\left[p\left(x_{0}, x\right)-p(x, x)\right] \\
\leq & 2\left[p\left(x_{0}, x\right)-\min \left\{p\left(x_{0}, x_{0}\right), p(x, x)\right\}\right] .
\end{aligned}
$$

Hence $h$ is uniformly continuous on $X$. Also,

So

$$
h\left(x_{0}\right)+h(x) \geq 2 p\left(x_{0}, x\right)-\lim _{n \rightarrow \infty} 2 p\left(x_{n}, x_{n}\right) .
$$

$$
h\left(x_{0}\right)-h(x) \geq p\left(x_{0}, x\right)+\frac{1}{2}\left[h\left(x_{0}\right)-3 h(x)\right]-\lim _{n \rightarrow \infty} p\left(x_{n}, x_{n}\right) .
$$

Now as $x=x_{m}$, we have

$$
\begin{aligned}
h\left(x_{0}\right)-h(x) \geq & p\left(x_{0}, x\right)-p\left(x_{m}, x_{m}\right)+\left[p\left(x_{m}, x_{m}\right)-\lim _{n \rightarrow \infty} p\left(x_{n}, x_{n}\right)\right] \\
& +\frac{1}{2}\left[h\left(x_{0}\right)-3 h(x)\right]
\end{aligned}
$$

so that as $m$ becomes large, we get $h\left(x_{m}\right) \rightarrow 0$ as $m \rightarrow \infty$. Hence

$$
h\left(x_{0}\right)-h(x) \geq p\left(x_{0}, x\right)-p(x, x)
$$

when $x=x_{m}, m$ is large, which further implies that $x_{0}$ is not $p$ - $h$ point.

\section{Application in fixed point theory}

Let $(X, p)$ be a partial metric space and $h$ be as given. Define $\ll$ on $X$ by the rule that for $x, y \in X$,

$x \ll y$ if and only if $h(y)-h(x) \geq p(x, y)-p(x, x)>0$.

Then $x \ll y$ relation orders $X$. The relation is transitive and antisymmetric.

Definition 3.1. A point $x_{0} \in X$ is said to be a minimal point with respect to $\ll$ if and only if $x \ll x_{0}$ implies $x=x_{0}$.

Theorem 3.2. A point $x_{0}$ in $X$ is $p$ - $h$ point for $h$ if and only if $x_{0}$ is a minimal point with respect to $\ll$.

Proof: If a point $x_{0} \in X$ is a $p$ - $h$ point for $h$, then

$$
h\left(x_{0}\right)-h(x)<p\left(x_{0}, x\right)-p(x, x)
$$

for all $x \in X$ with $x \neq x_{0}$. This gives $x \ll x_{0}$ only if $x=x_{0}$. Therefore $x_{0}$ is a minimal point with respect to $\ll$.

Conversely, let $x_{0}$ be a minimal point with respect to $\ll$, so $\mathrm{x} \ll x_{0}$ implies $x=x_{0}$, i.e., if $x \neq x_{0}$, then

$$
h\left(x_{0}\right)-h(x)<p\left(x_{0}, x\right)-p(x, x)
$$

for all $x \in X$ with $x \neq x_{0}$. This implies $x_{0}$ is a $p$-h point for $h$.

Theorem 3.3. Given a function $f: X \rightarrow X$, it may be possible to take a partial metric $p$ and a function $h$ so that $\ll$ has the property $f(x) \ll x$. Then any $p$-hpoint for $h$ is a fixed point of $f$.

Proof. Let $x_{0}$ be a $p$-hpoint for $h$, then by Theorem 3.2, $x_{0}$ is a minimal point with respect to $\ll$. Again by hypothesis $f\left(x_{0}\right) \ll x_{0}$. It then follows that $f\left(x_{0}\right)=x_{0}$, a fixed point of $f$. 


\section{A.P. Baisnab and Sumana Pal}

\section{Some applications of $p$-hpoints in a metric space}

Theorem 4.1. (Kannan Fixed Point Theorem)

Let $(X, d)$ be a complete metric space and $f: X \rightarrow X$ satisfies

$$
d(f(x), f(y)) \leq \alpha[d(x, f(x))+d(y, f(y))]
$$

for all $x, y \in X$ where $0 \leq \alpha<\frac{1}{2}$, and let $d(x, f(x))$ be a 1.s.c. function, then $f$ has a fixed point in $X$.

Proof. We take

$h(x)=\frac{1-\alpha}{1-2 \alpha} d(x, f(x))$.

Then $h: X \rightarrow \mathbb{R}$ is al.s.c. function being bounded below. In view of Theorem 2.3, we find that there is a $d$ - $h$ point in $X$.

Now

Again,

$$
h(x)-h(f(x))=\frac{1-\alpha}{1-2 \alpha}\left[d(x, f(x))-d\left(f(x), f^{2}(x)\right)\right] .
$$

which implies

$$
d\left(f(x), f^{2}(x)\right) \leq \alpha\left[d(x, f(x))+d\left(f(x), f^{2}(x)\right)\right]
$$

Hence

$$
d\left(f(x), f^{2}(x)\right) \leq \frac{\alpha}{1-\alpha} d(x, f(x)) .
$$

$$
\begin{gathered}
h(x)-h(f(x)) \geq \frac{1-\alpha}{1-2 \alpha}\left[d(x, f(x))-\frac{\alpha}{1-\alpha} d(x, f(x))\right] \\
=\left(\frac{1-\alpha}{1-2 \alpha}\right)\left(\frac{1-\alpha-\alpha}{1-\alpha}\right) d(x, f(x)) \\
\geq d(x, f(x)) .
\end{gathered}
$$

Therefore $f(x) \ll x$. Now we apply Theorem 3.3 to conclude that $f$ has a fixed point in $X$.

Theorem 4.2. (B. Fisher Theorem)

Let $(X, d)$ be a complete metric space and $f: X \rightarrow X$ satisfies

$$
d(f(x), f(y)) \leq \alpha[d(x, f(y))+d(y, f(x))]
$$

for all $x, y \in X$, where $0 \leq \alpha<\frac{1}{2}$ and let $d(x, f(x))$ be a 1.s.c. function, then $f$ has a fixed point in $X$.

Proof: Here also we take

Then

$$
h(x)=\frac{1-\alpha}{1-2 \alpha} d(x, f(x)) .
$$

Now

$$
h(x)-h(f(x))=\frac{1-\alpha}{1-2 \alpha}\left[d(x, f(x))-d\left(f(x), f^{2}(x)\right)\right] .
$$

$$
\begin{gathered}
d\left(f(x), f^{2}(x)\right) \leq \alpha\left[d\left(x, f^{2}(x)\right)+d(f(x), f(x))\right] \\
\leq \alpha\left[d(x, f(x))+d\left(f(x), f^{2}(x)\right)\right]
\end{gathered}
$$

$$
d\left(f(x), f^{2}(x)\right) \leq \frac{\alpha}{1-\alpha} d(x, f(x)) .
$$


On $p$ - $h$ Points and Completeness Property of a Partial Metric Space

Hence

$$
h(x)-h(f(x)) \geq d(x, f(x)) .
$$

Thus $f(x) \ll x$. Since by Theorem 2.3, we get a $d$-h of $h$ in $X$, we find that there exists a fixed point of $f$ in $X$.

Theorem 4.3. Let $(X, d)$ be a complete metric space and $f: X \rightarrow X$ satisfies

$$
\begin{gathered}
d(f(x), f(y)) \leq \alpha[d(x, f(x))+d(y, f(y))]+\beta d(x, y) \\
+\gamma \max \{d(x, f(y)), d(y, f(x))\}
\end{gathered}
$$

for all $x \in X$ where $\alpha, \beta, \gamma \geq 0,2 \alpha+\beta+2 \gamma<1$, and let $d(x, f(x))$ be a 1.s.c. function, then $f$ has a fixed point in $X$.

Proof. Here we take $h(x)=a d(x, f(x))$, for all $x \in X$, where $a=\frac{1-\alpha-\gamma}{1-2 \alpha-\beta-2 \gamma}$.

Then

Now

$$
h(x)-h(f(x))=a\left[d(x, f(x))-d\left(f(x), f^{2}(x)\right)\right] .
$$

$$
\begin{gathered}
d\left(f(x), f^{2}(x)\right) \leq \alpha\left[d(x, f(x))+d\left(f(x), f^{2}(x)\right)\right]+\beta d(x, f(x)) \\
+\gamma \max \left\{d\left(x, f^{2}(x)\right), d(f(x), f(x))\right\} \\
\leq \alpha\left[d(x, f(x))+d\left(f(x), f^{2}(x)\right)\right]+\beta d(x, f(x))+\gamma d\left(x, f^{2}(x)\right) \\
\leq(\alpha+\beta+\gamma) d(x, f(x))+(\alpha+\gamma) d\left(f(x), f^{2}(x)\right) .
\end{gathered}
$$

This gives

which implies

$$
d\left(f(x), f^{2}(x)\right) \leq \frac{\alpha+\beta+\gamma}{1-\alpha-\gamma} d(x, f(x))
$$

$$
h(x)-h(f(x)) \geq a\left[1-\frac{\alpha+\beta+\gamma}{1-\alpha-\gamma}\right] d(x, f(x))=d(x, f(x)) .
$$

Hence $f(x) \ll x$. Then we proceed as before to complete the proof.

\section{Conclusions}

In this paper, a kind of characterization of completeness property of a partial metric space has been achieved, by using the notion of $p$ - $h$ point relevant to fixed point theory in the space.

Acknowledgement. The authors remain thankful to the reviewer for the kind and valuable advice for improvement of the paper in the present form.

\section{REFERENCES}

1. A.Sindersiya, A.Pariya, N. Gupta and V. H. Badshah, common fixed point theorem in complex valued metric spaces satisfying rational inequality, Annals of Pure and Applied Mathematics, 12 (2) (2016) 137-142.

2. B.S.Choudhury,A.Kundu and N.Metiya, Fixed point results for Ciric type weak contraction in metric spaces with applications to partial metric spaces, Filomat, 28 (7) (2014) 1505-1516.

3. B.S.Choudhury and C.Bandyopadhyay, Suzuki type common fixed point theorem incomplete metric space and partial metric space, Filomat, 29 (6) (2015) 1377-1387. 


\section{A.P. Baisnab and Sumana Pal}

4. B.K.Lahiri, P.Das and L.K.Dey, Cantor's theorem in 2-metric spaces andits applications to fixed point problems, Taiwanese J. Math., 15 (1) (2011) 337 - 352.

5. D.Dhamodharan and R. Krishnakumar, Cone S-metric space and fixed point theorems of contractive mappings, Annals of Pure and Applied Mathematics, 14(2) (2017) 237-243.

6. I.Altun and S.Romaguera, characterizations of partial metric completeness in terms of weakly contractive mappings having fixed point, Appl. Discrete Math., 6 (2012) 247 - 256.

7. J.D.Weston, A characterization of metric completeness, Proc. Amer. Math. Soc., 64(1) (1977) 186 - 188.

8. N.Shioji, T.Suzuki and W.Takahashi, Contractive mappings, Kannan mappings and metric completeness, Proceedings of the Amer. Math. Society, 126 (10) (1998) 31173124.

9. O.Valero, On Banach fixed point theorems for partial metric spaces, Applied General Topology, 6(2) (2005) 229240.

10. P.Das and L.K.Dey, Fixed point of contractive mappings in generalized metric spaces, Math. Slovaca, 59 (4) (2009) 499 - 504.

11. P.Pathak, A.Pariya, V.H.Badshah and N.Gupta, Fixed point theorems for Kannan contractions and weakly contractive mappings on a modular metric space endowed with a graph, Annals of Pure and Applied Mathematics, 14 (1) (2017) 77-85.

12. R.Kannan, Some results on fixed points, Bull. Cal. Math. Society, 60 (1968) 71-76.

13. R.Kannan, Some results on fixed points II, Amer. Math. Monthly, 76 (1969) 405 408.

14. S.G.Matthews, Partial metric topology, Proc. 8th Summer Conference on GeneralTopology and Applications, Ann. New York Acad. Sci., 728 (1994) 183-197.

15. S.Oltra and O.Valero, Banach's fixed point theorem for partial metric spaces, Rendicontidell Istituto di Matematica dell Universita di Trieste, 36 (2004) 17-26.

16. S.Romaguera, A Kirk type characterization of completeness for partial metric spaces, Fixed Point Theory and Applications, Article ID 493298 (2010), 6 pages.

17. T.Senapati, L.K.Dey and D.Dolianineki, Extensions of Ćirić and Wardowski type fixed point theorems in D-generalized metric spaces, Fixed Point Theory Appl., Paper No. 33 (2016) 14 pp. 